\title{
Backlash Error Measurement and Compensation on the Vertical Machining Center
}

\author{
Huanlao Liu*, Xiaoning Xue, Guangyu Tan \\ Engineering college, Guangdong Ocean University, Zhanjiang, China \\ E-mail: hl66@163.com, \{xuexn,tangy\}@gdou.edu.cn \\ Received December 21, 2009; revised February 9, 2010; accepted February 18, 2010
}

\begin{abstract}
The position errors in the axis direction of a vertical machine center have been measured by means of the VM101 linear encored measurement system. The character of the backlash error is discussed; results show that the backlash error has great influence on the position error. The position accuracy is enhanced after the backlash errors are compensated.
\end{abstract}

Keywords: Position Error, Backlash Error, Error Model, Artificial Neural Network

\section{Introduction}

The laser interferometer is commonly used in the measurement of various error components. Geometric errors have many different components like linear displacement error (positioning accuracy), straightness and flatness of movement of the axis, spindle inclination angle, square ness error, backlash error etc. The errors can be reduced with the structural improvement of the machine tool through better design and manufacturing practices. However, in most cases, due to physical limitations production and design techniques cannot solely improve the machine tool accuracy. Therefore, identification, characterization and compensation of these error sources are necessary to improve machine tool accuracy cost-effectively. EungSuk Lee et al. [1] used the interferometer in measuring 19 of the 21 components on a three-axis CNC milling machine with the exception of angular roll. Ni et al. [2] developed an optical system consisting of a laser interferometer, beam splitters, flat mirrors and dual-axis lateraleffect photo detectors to facilitate simultaneous on-line measurement of five errors on each axis, which are linear and vertical straightness, roll, pitch and yaw errors. Shin [3], Ertekin et al. [4] also presented the results of accuracy characterization of a CNC Machining Center using laser interferometer. Other techniques such as disc checks [5] and double ball bar (DBB) [6] measurements are also used in the position error measurement. Knapp et al. [7] listed a few tests for assessing the quality of machine tools on a periodic basis. The diagonal displacement test was one of the means adopted to estimate the volumetric accuracy of the machine tool through evaluation of the positioning accuracy and repeatability along the body diagonals. The circular test can also be used in assessing the accuracy of the machine tools and the identification of backlash error, a software strategy that is able to compensate the backlash error [8].

Backlash error can be obtained through some kinds of methods, it is either time-costly (laser interferometer) or only the maximum value obtained (DBB) and the effects of backlash error on the machine accuracy are not discussed in detail so far. The aim of the investigation presented in this paper was to illustrate the effects of the backlash error on the position accuracy of the machine tools and attracted more attention about the backlash error in the machine production process.

VM101 Comparator System, which has the advantages of easy operation, time-less and also high accuracy, was used to measure the axis linear displacement and backlash error in this paper. A simple introduction about the system and measurement process was given in the following section. The third part presents the experimental results. Two kinds of models including Back Propagation Artificial Neural Network (BP-ANN) and polynomial function model that can be used in different occasions were presented in the fourth part. The results after backlash error compensated were given in the fifth part. Then some conclusions have made in the final part of this paper.

\section{Experimental Procedures}

\subsection{The VM101 Linear Encored Measurement System}

The VM101 Comparator System which has made in 
HEIDENHAIN cooperation consists of a precision scanning head, auxiliary carriage, interface electronics and sturdy storage case. The sturdy scale carrier is a U-shaped stainless steel extrusion. The material measure-a precision scale with a phase grating graduation and an $8 \mu \mathrm{m}$ grating period-lies in the neutral stress zone of the U-shaped scale carrier. The scanning head which is usually connected to the spindle of the machine tools runs along the scale without making mechanical contact. It is an exposed incremental linear encoder with high accuracy. Its measuring lengths is $720 \mathrm{~mm}$, accuracy grade is $\pm 1 \mu \mathrm{m}$ at $20^{\circ} \mathrm{C}\left( \pm 0.00004\right.$ in. at $\left.68^{\circ} \mathrm{F}\right)$ [9]. Figure 1 shows the measurement setup on the $\mathrm{X}$-axis.

\subsection{Measurement Process}

A new vertical machining centre tape DM4600 was chosen for the tests. Its working volume was $600 \times 400 \times$ 420 (mm). After the workspace was defined, accuracy and repeatability were tested for each axis direction. To minimize the error that might arise due to the temperature change during the measurements because of the axis motions. The machine was put in operation for three hours with a programmed exercise. This routine activated all the axis motions simultaneously before any measurements have been taken so that the machine could pass the thermal transition state to reach its equilibrium state.

In order to keep errors resulting from uneven guide ways (Abbe error) at a minimum, the comparator system must be installed as near as possible both to the point of tool contact as well as to the encoder. The sides of the scale carrier should be aligned with the axis to be measured within $0.1 \mathrm{~mm}$.

To eliminate the influence of speeds, the machine was programmed to move with a lower feed rate of $50 \mathrm{mmpm}$, after the warm-up cycle, position displacement measurements were taken in both travel directions. The data was captured at equal intervals $(20 \mathrm{~mm})$ and the measurement cycle $\mathrm{CNC}$ part program composes a sequence of moves beginning at one limit of the axis in forward direction ( $\mathrm{f}$ ), extending to the opposite limit, and returning to the beginning position in reverse direction(r). The commanded machine position is nominal axis position, which is determined by the part program being executed. A PC reads the absolute machine positions directly into

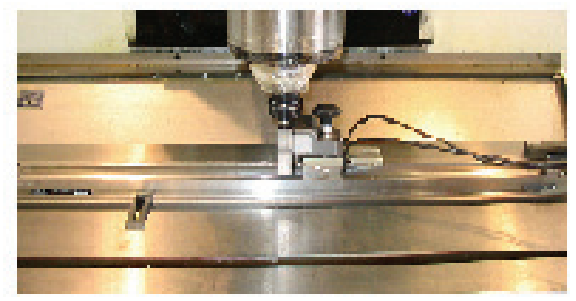

Figure 1. The $\mathrm{X}$-axis measurement setup. the database from the VM101. A sample measurement cycle CNC program for X-axis is given in Table 1 .

\section{Experimental Results}

\subsection{Axis Linear Displacement Error}

The X-axis linear displacement error plots are shown in Figure 2. In the legend of the error graphs, Error 1-f and Error 1-r indicates that errors were measured when the machine was in the first cycle motion for forward (f) and reverse (r) directions after about 3 hours warm-up period. The error plots show that positional errors are generally lower at the beginning of axis travel (home position) and have linearly increasing trend with increasing axis nominal position. It was found that the position error in forward direction was bigger than the position error in the reverse direction at the same point. The maximum displacement error is about $40 \mu \mathrm{m}$ at the end of the axis.

The Y-axis linear displacement error (Figure 3) and the Z-axis linear displacement error (Figure 4) showed similar linear trends with increasing nominal axis position as in $\mathrm{X}$-axis. The $\mathrm{Y}$-axis linear displacement error was the smallest among the three axes being tested and the magnitude was approximately $25 \mu \mathrm{m}$. Maximum linear displacement error of the Z-axis was almost the same value as the end of the $\mathrm{X}$-axis. The slope of the error line of the $\mathrm{Y}$-axis is the smallest when the machine is moving in the forward directions. There are obviously two

Table 1. VM101 measurement cycle CNC program.

\begin{tabular}{rr}
\hline N001 \#1 =0 & N010 X560 \\
\hline N002 G92 X0 Y0 Z0 & N011 G04 P10 \\
N003 WHILE \#1 LT 28 & N012 WHILE \#1 LT 20 \\
N004 G91 G01 X20 F50 & N013 G91 G01 X-20 \\
N005 G04 P10 & N014 G04 P10 \\
N006 \#1 = \#1 + 1 & N015 \#1 = \#1 + 1 \\
N007 ENDW & N016 ENDW \\
N008 \#1 =0 & N017 M30 \\
N009 G90 G01 X562
\end{tabular}

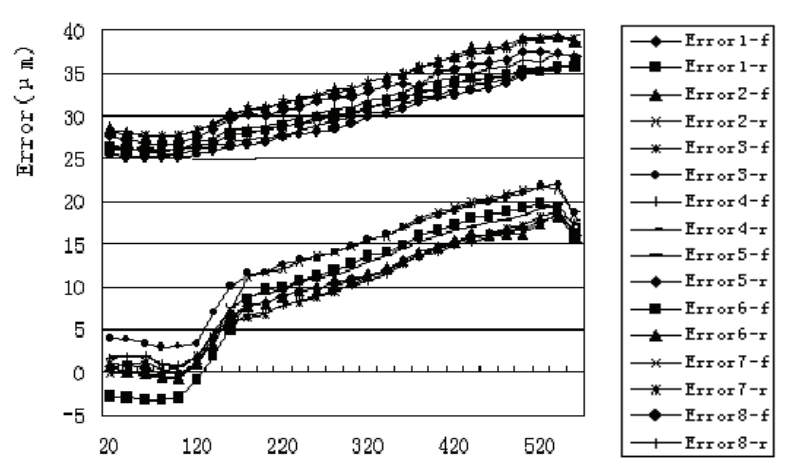

X-Axis Nominal Position (mm)

Figure 2. X-axis linear displacement error. 


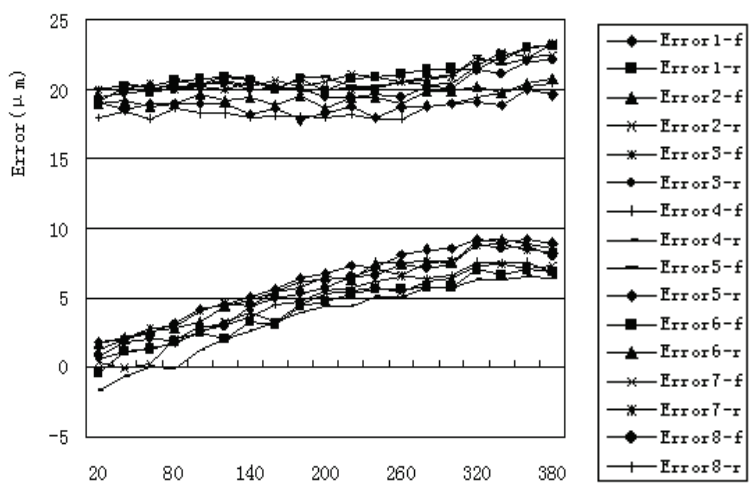

Y-Axis Nominal Position (mm)

Figure 3. Y-axis linear displacement error.

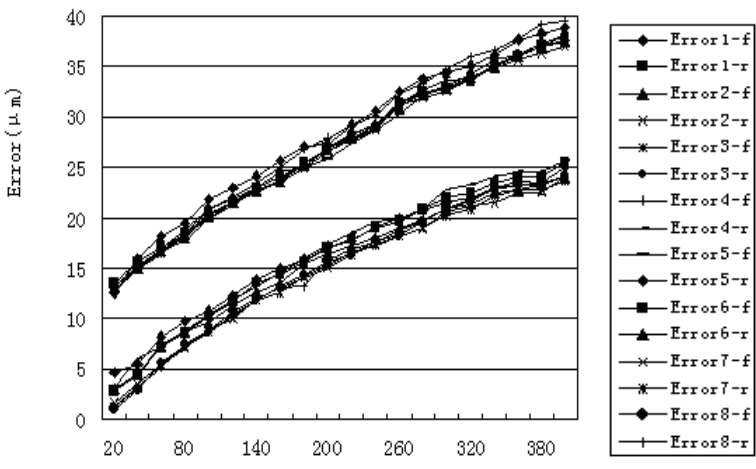

Z-Axis Nominal Position (mm)

Figure 4. Z-axis linear displacement error.

groups error line on the axis linear displacement error plot. That means there are two different error values at the same location in the axis. The main reason of these results is the backlash error.

\subsection{Backlash Error}

Backlash error is a position dependent error affecting the contouring accuracy. When the axis changes direction from one side to the other, there is a lag before the table starts moving again, that would cause position errorbacklash error. The backlash error was the major reason why there are two groups of error lines on the displacement error plot. It has great influence on the accuracy of the machine and should be compensated. Figure 5 shows the backlash error of the axes. $B_{e x}$ means the X-axis backlash error, $B_{e y}$ is the $\mathrm{Y}$-axis backlash error and the $\mathrm{Z}$-axis backlash error is $B_{e z}$.

The backlash error value of the axis was not constant and has different value at different axis nominal positions. Maximum backlash error is about $27 \mu \mathrm{m}$ at the beginning of travel in X-axis, the backlash error is $18 \mu \mathrm{m}$ and $11 \mu \mathrm{m}$ at the home point of the $\mathrm{Y}$-axis and $\mathrm{Z}$-axis. It leads to the relatively large error of the axis in the forward direction. The Z-axis has the smallest average

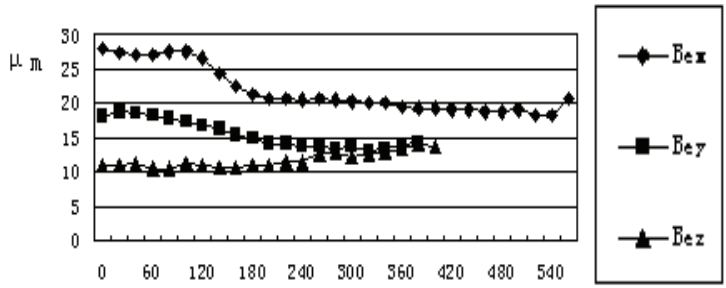

Axis Nominal Position (mm) Figure 5. The value of backlash error.

backlash error among the three axes and obtained maximum $B_{e z}$ at the end of the axis travel with a magnitude of $14 \mu \mathrm{m}$.

\section{The Models of Backlash Error}

\subsection{The ANN Models of Backlash Error}

The neural net using the back-propagation algorithm has been used to predict the total positioning error at any given thermal state and location of the cutting tool from knowledge of that error at some specified point in the workspace as measured by the laser ball bar [10]. The neural network which utilizes the learning ability to predicate the backlash error value from the knowledge of the error as measured by the VM101 system was developed. The neural networks developed in this study have three layers-one input layer, one hidden layer, and one output layer. The input layer comprises the units that represent the nominal position of the axis. The output layer has three output units that represent the backlash errors of the three axes. The hidden units of the network are used to extract the underlying features of relationships between the nominal position and the backlash errors of the machine tools. The Figure 6 shows the flow chart of the neural net work.

Training of the networks can proceed only after the entire experimental data is obtained. The training process of the net was shown in Figure 7.

During learning, the nominal position value of the axis was input into the self-learning and prediction unit, the output of the neural net is the backlash error value. Then the backlash error compensation can be taken. However because the training time is relatively long, the ANN model can only be used in the off-line compensation, the advantage of the net model was very high-nonlinear mapping ability.

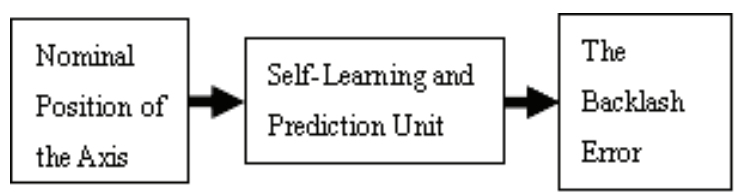

Figure 6. The flow chart of the neural net. 


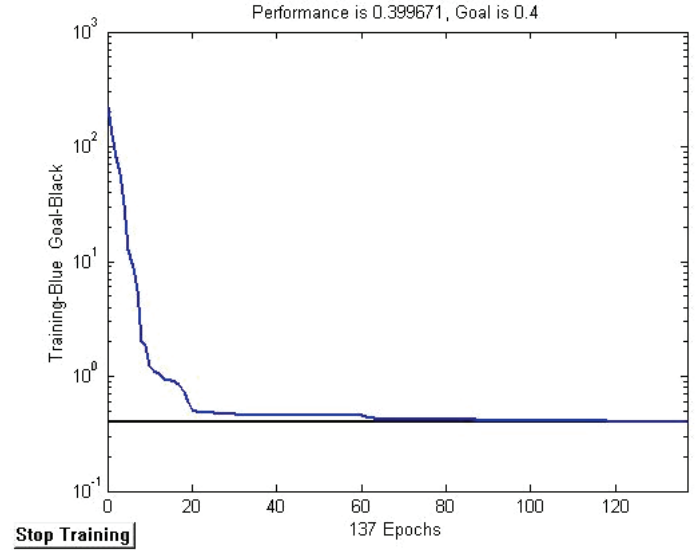

Figure 7. The training process of the neural network.

\subsection{The Polynomial Models of Backlash Error}

For on-line compensation, the backlash errors $\left(B_{e x}, B_{e y}, B_{e z}\right)$ can also be modeled as polynomial functions with the position along each axis. As the Equations (1), (2) and (3) expressed.

$$
\begin{aligned}
& B_{e x}=a_{0}+a_{1} x+a_{2} x^{2}+a_{3} x^{3} \\
& B_{e x}=b_{0}+b_{1} y+b_{2} y^{2}+b_{3} y^{3} \\
& B_{e x}=c_{0}+c_{1} z+c_{2} z^{2}+c_{3} z^{3}
\end{aligned}
$$

The error models then can identify using the leastsquares criterion method. The calculation process of the backlash error value was very fast, but the drawback of the polynomial model was its relatively lower accuracy.

\section{Accuracy after Backlash Error Compensated}

After the models have been developed, the backlash error then can be compensated. The procedure can be divided into three stages: calibration of the backlash errors (part 2 ), modeling of the errors (part 4), and compensation control implementation.

The principle of the error compensation control implementation is as the following steps: the backlash errors on the nominal position of the axis at any given thermal stage are collected and the errors are estimated by the models. Then the resultant errors between the commanded position and the real position are calculated. Finally, the compensation singles are sent to the CNC controller to shift the origins of the slide axes to implement the error compensation. The results of the position accuracy after the backlash error compensated using the ANN model were measured using the same procedure as mentioned above. Figures 8-10 show the X-axis, Y-axis and $\mathrm{Z}$-axis measurement results.

All three plots show that the backlash errors were well compensated and the accuracy of these axes was highly increased. All linear positional errors increasing linearly with respect to axis nominal position and are the highest at the end of the axis travel range. These results correspond with the most previous work and the assumption that the linear errors components change linearly with position. Machine home position gave best linear accuracy; maximum linear displacement error was about $10 \mu \mathrm{m}$ in the $\mathrm{X}$ and $\mathrm{Y}$-axes, the $\mathrm{Z}$-axis maximum linear displacement was about $25 \mu \mathrm{m}$ at the end of the axis position.

The variation of the linear displacement error (position systematic deviation) was about $\pm 2 \mu \mathrm{m}$ at the same position in the axis when the backlash errors were compensated. The repeatability of position of the axes was obtained with a magnitude of about $4 \mu \mathrm{m}$. This is the limits

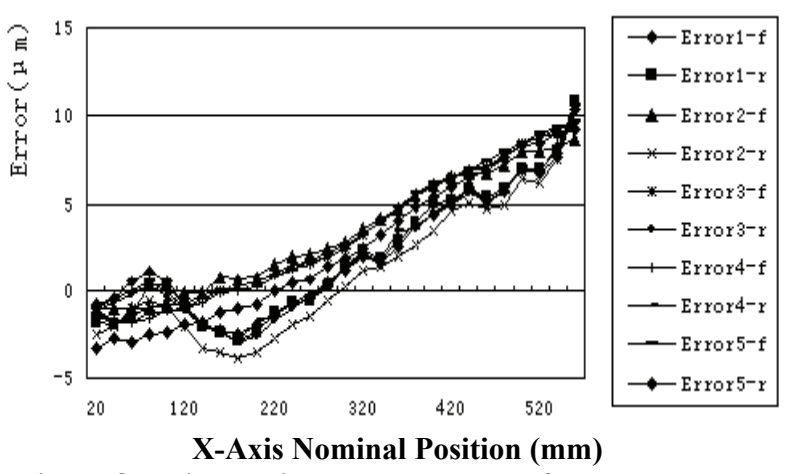

Figure 8. $X$ linear displacement error after compensated.

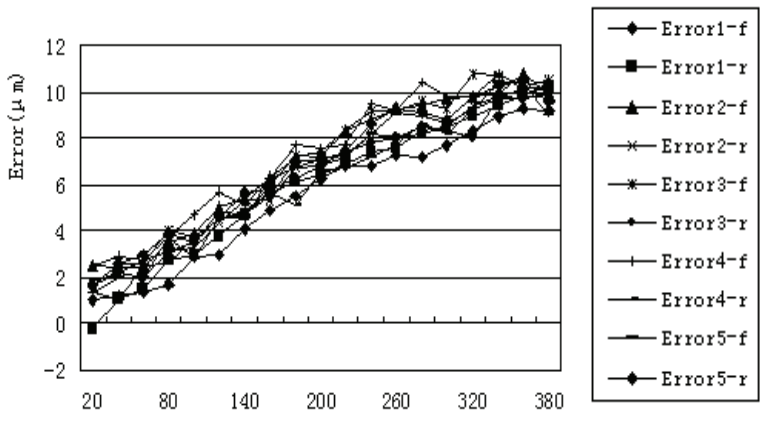

Y-Axis Nominal Position (mm)

Figure 9. Y linear displacement error after compensated.

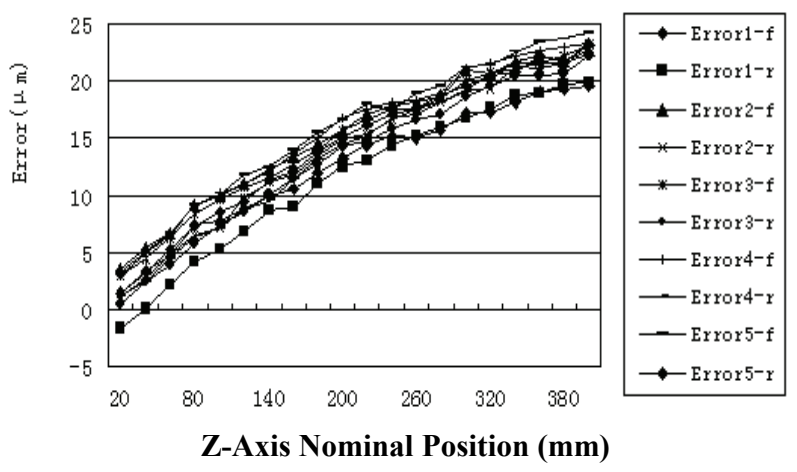

Figure 10. $\mathrm{Z}$ linear displacement error after compensated. 
accuracy through the off-line software for the softwarecompensation.

The experimental results show that the accuracy was enhanced after the backlash error eliminated. So more attention should be paid to the backlash error during the production and assemble processing of the machine tools.

\section{Conclusions}

From the results of this research, the following conclusions can be drawn:

- All linear position errors increased almost linearly with respect to axis nominal position and are the highest at the end of the axis travel range even the backlash error compensated.

- The backlash errors cause relatively large linear displacement error deviation between different directions at the same point in the axis. The backlash errors value is the function of the point in the axis.

- The accuracy of the machine increased after the backlash error compensated, so more attentions should be paid to the backlash errors in the machine tool production procession.

\section{Acknowledgements}

This work was financially supported for the research of geometric error measurement and compensation on $\mathrm{CNC}$ machine tool by National 863 Subject Plan under grant 2002AA423260.

\section{References}

[1] E. S. Lee, S. H. Suh and J. W. Shon, "A Comprehensivemethod for Calibration of Volumetric Positioning Accuracy on CNC Machines," The International Journal of Advanced Manufacturing Technology, Vol. 14, No. 1, 1998, pp. 43-49.
[2] J. Ni and S. M. Wu, "An On-Line Measurement Technique for Machine Volumetric Error Compensation," Journal of Engineering for Industry, Vol. 115, 1993, pp. $85-92$.

[3] Y. C. Shin, H. Chin and M. J. Brink, "Characterization of CNC Machining Centers," Journal of Manufacturing Systems, Vol. 10, No. 5, 1992, pp. 407-421.

[4] Y. M. Ertekin and A. C. Okafor, "Vertical Machining Center Accuracy Characterization Using Laser Interferometer, Part 1. Linear Positional Errors," Journal of Material Processing Technology, Vol. 105, No. 3, 2000, pp. 394-406.

[5] W. Knapp, "Test of the Three-Dimensional Uncertainty of Machine Tools and Measuring Machine and its Relation to the Machine Errors," Annals of CIRP, Vol. 32, No. 1, 1983, pp. 459-464.

[6] Y. Kakino, Y. Ihara and Y. Nakatsu, "The Measurement of Motion Errors of NC Machine Tools and Diagnosis of their Origins by Using Telescoping Magnetic Ball Bar Method," Annals of the CIRP, Vol. 36, No. 1, 1987, pp. 377-380.

[7] W. Knapp, "Interim Checks for Machine Tools," Proceedings of the 3rd International Conference on Laser Metrology and Machine Performance-LAMDAMP, 1997, pp. 161-168.

[8] Y. S. Tarng, J. Y. Kao and Y. S. Lim, "Identification of and Compensation for Backlash on the Contouring Accuracy of CNC Machine Center," The International Journal of Advanced Manufacturing Technology, Vol. 13, No. 2, 1997, pp. 359-366.

[9] "HEIDENHAIN Mounting and Operating InstructionsVM 101 Incremental Comparator System," Germany, 2000.

[10] N. Srinnivasa, J. C. Ziegert and S. Smith, "Prediction of Position Errors of a Three Axis Machine Tool Using a Neural Network," Proceedings of the Japan/USA Symposium on Flexible Automation, San Francisco, 1992, pp. 203-209. 